\title{
The global prevalence of latent tuberculosis: a systematic review and meta-analysis
}

\author{
Adam Cohen (10) ${ }^{1,8}$, Victor Dahl Mathiasen (10) $2,3,8$, Thomas Schön ${ }^{4,5}$ and \\ Christian Wejse $3,6,7$
}

Affiliations: ${ }^{1}$ Dept of Pathology, St Olavs Hospital, Trondheim, Norway. ${ }^{2}$ International Reference Laboratory of Mycobacteriology, Statens Serum Institut, Copenhagen, Denmark. ${ }^{3}$ Dept of Infectious Diseases, Aarhus University Hospital, Aarhus, Denmark. ${ }^{4}$ Division of Microbiology and Molecular Medicine, Dept of Clinical and Experimental Medicine, Linköping University, Linköping, Sweden. ${ }^{5}$ Dept of Clinical Microbiology and Infectious Diseases, Kalmar County Hospital, Kalmar, Linköping University, Linköping, Sweden. ${ }^{6}$ Bandim Health Project, INDEPTH Network, Bissau, Guinea-Bissau. ${ }^{7}$ Center for Global Health, Aarhus University (GloHAU), Aarhus, Denmark. ${ }^{8}$ Both authors contributed equally.

Correspondence: Christian Wejse, Dept of Infectious Diseases, Aarhus University Hospital, Aarhus N, 8200, Denmark. E-mail: wejsedadadlnet.dk

@ERSpublications

Using a novel strategy, global estimates of latent tuberculosis were updated to $24.8 \%$ for IGRAs and 21.2\% for TSTs using prevalence surveys of 351811 individuals. Regional estimates varied between 11-27\% and 12-33\% for IGRAs and TSTs respectively. http://bit.ly/2MN8Gvu

Cite this article as: Cohen A, Mathiasen VD, Schön T, et al. The global prevalence of latent tuberculosis: a systematic review and meta-analysis. Eur Respir J 2019; 54: 1900655 [https://doi.org/10.1183/ 13993003.00655-2019].

ABSTRACT In 1999, the World Health Organization (WHO) estimated that one-third of the world's population had latent tuberculosis infection (LTBI), which was recently updated to one-fourth. However, this is still based on controversial assumptions in combination with tuberculin skin test (TST) surveys. Interferon- $\gamma$ release assays (IGRAs) with a higher specificity than TST have since been widely implemented, but never used to estimate the global LTBI prevalence.

We conducted a systematic review and meta-analysis of LTBI estimates based on both IGRA and TST results published between 2005 and 2018. Regional and global estimates of LTBI prevalence were calculated. Stratification was performed for low, intermediate and high TB incidence countries and a pooled estimate for each area was calculated using a random effects model.

Among 3280 studies screened, we included 88 studies from 36 countries with 41 IGRA ( $\mathrm{n}=67$ 167) and 67 TST estimates $(n=284644)$. The global prevalence of LTBI was $24.8 \%$ (95\% CI 19.7-30.0\%) and $21.2 \%$ (95\% CI 17.9-24.4\%), based on IGRA and a 10-mm TST cut-off, respectively. The prevalence estimates correlated well to WHO incidence rates ( $\mathrm{Rs}=0.70, \mathrm{p}<0.001)$.

In the first study of the global prevalence of LTBI derived from both IGRA and TST surveys, we found that one-fourth of the world's population is infected. This is of relevance, as both tests, although imperfect, are used to identify individuals eligible for preventive therapy. Enhanced efforts are needed targeting the large pool of latently infected individuals, as this constitutes an enormous source of potential active tuberculosis.

This article has supplementary material available from erj.ersjournals.com

Data available from the Dryad Digital Repository: https://doi.org/10.5061/dryad.9r1h22d

Received: 01 April 2019 | Accepted after revision: 11 June 2019

Copyright OERS 2019 


\section{Introduction}

The World Health Organization (WHO) estimated in 1999 that 1.8 billion people, or one-third of the world's population, were infected with Mycobacterium tuberculosis, but without clinical symptoms of active tuberculosis (TB), which is the definition of latent TB infection (LTBI) [1]. This estimate has since been referred to frequently, but had not been updated until recently. In 2016, a WHO-endorsed estimate updated the global prevalence of LTBI to $23 \%$, corresponding to 1.7 billion people infected worldwide [2, 3].

The reactivation rate of LTBI into active disease is controversial, partly as re-infection may occur, but mainly because there are no methods to identify LTBI subjects at highest risk of developing active TB. Nevertheless, the current estimate of the LTBI burden clearly indicates a large reservoir of individuals at risk of developing active TB. Global incidence and mortality rates of active TB have declined since 1990, and the global incidence rate has been decreasing since the WHO goals were appointed at the beginning of the new millennium [3]. Improved attention to LTBI screening and preventive therapy has been pointed out as crucial for the End TB Strategy for 2050 to be achieved $[4,5]$. It is hardly possible to eliminate TB unless progression to active $\mathrm{TB}$ is prevented, underlining the need to determine the actual prevalence of LTBI and define hot-spot areas [6].

The previous WHO estimate was only based on tuberculin skin test (TST) to a small extent (13\%) [1], but mainly on annual risk of infection calculated from the incidence of smear-positive cases using the STYвLO [7] rule, derived from empirical data and assumptions on duration of infectiousness and transmissions per year. The ratio assumes that each smear-positive case transmits 10 infections per year; whereas a newer estimate suggested that this number could be as low as two to six infections [8]. In addition, the Styblo rule assumes that the duration of infectiousness is in general 2 years, which is now debated and likely to have decreased due to intensified case finding and treatment of active TB. Even in resource-poor settings, treatment delay is reduced to an average of 3 months, which enables a more rapid sputum conversion and reduction in infectiousness than when the rule was defined $[9,10]$. Additionally, transmission rates and infectiousness are highly dependent on age distributions, geographical location, drug availability, living conditions and population density [8]. Therefore, the assumption that the Styblo rule, even in its revised form used for the recent update, still applies in the global TB settings of today could lead to an overestimation of LTBI prevalence. Hence, basing LTBI prevalence on a rule of thumb, involving assumptions which may not be valid today, is likely to be more imprecise than using real data collected on populations in a large number of countries which do not involve assumptions on transmission rates or infectiousness, but incorporate local conditions.

TST has traditionally been used as a screening tool for LTBI due to low direct costs and ease of use. In recent decades, commercial interferon $-\gamma$ release assays (IGRAs) have been introduced, which solely contain antigens that are absent in bacille Calmette-Guérin (BCG) vaccine strains and require no follow-up test [11]. Thus, IGRAs have superior specificity to TST in BCG-vaccinated populations and in regions with frequent nontuberculous mycobacteria exposure [12].

So far, no global estimation of LTBI prevalence has been based on IGRAs, and it has been suggested that the prevalence of LTBI might be overestimated by TST compared with IGRA due to the improved specificity [13]. In this study, we aimed to investigate the global prevalence of LTBI based on TB incidence-stratified estimates directly derived from both IGRA and TST, as these are the tests currently applied to identify individuals for preventive therapy.

\section{Methods}

Search strategy and selection criteria

We performed a systematic review and meta-analysis using the Preferred Reporting Items for Systematic Reviews and Meta-analyses (PRISMA) guidelines (supplementary material section F) [14]. The protocol was registered on PROSPERO (www.crd.york.ac.uk/prospero/; CRD42019125380).

Studies that reported on the prevalence of LTBI diagnosed with IGRA and/or TST were eligible. Two investigators (A. Cohen and V.D. Mathiasen) searched the databases MEDLINE, Embase, Scopus and Web of Science for articles published between January 1, 2005 and July 30, 2018 using the following terms in different combinations and constructions depending on the applied database: "latent tuberculosis" and ("tuberculosis" AND "prevalence" AND "latent") combined with "tuberculin test", "tuberculin tests", "tuberculin skin test”, "tuberculin skin tests", “TST”", "Mantoux ${ }^{\star ”, ~ " i n t e r f e r o n-g a m m a ”, ~}$ "interferon-gamma release assay", "interferon-gamma release assays", "interferon-gamma release test”, "interferon-gamma release tests", "IGRA*", "Quantiferon*”, "QFT*”, "T-SPOT.TB*”, "enzyme-linked immunospot assay" and "enzyme-linked immunospot assays" (detailed electronic search strategy available in supplementary material section A). All search terms were searched in both title, abstract and field keywords. Our search combined free text and subject identifiers as medical subject heading (MeSH) terms. 
Additionally, we examined several reference lists of relevant articles. We did not set any language restrictions. The search was initiated in 2005 reflecting the widespread availability of stable, quality-controlled and commercial IGRAs on the market, and in order to provide an updated analysis with recent surveys. The QuantiFERON-TB (QFT) test introduced in 2001 measured response to the same antigen mixture (purified protein derivate) as TST, while the QFT Gold (QFT-G) introduced in 2005 omitted antigens present in the BCG vaccine and in the ubiquitous nontuberculous mycobacteria.

Full texts were obtained for all studies identified by either A. Cohen or V.D. Mathiasen as potentially relevant.

\section{Study eligibility and quality assessment}

Following deletion of duplicates, A. Cohen and V.D. Mathiasen screened titles, abstracts or entire articles for exclusion criteria and determined which studies met the eligibility criteria (supplementary material section B). Only studies with a sample size of $\geqslant 200$ were included, to avoid selection bias from small studies. Meta-analyses, reviews, cost-effectiveness analyses and nonhuman studies were excluded. Studies on patients with presumed or active TB were excluded, as well as if they did not report on prevalence of LTBI using IGRA-tests (any versions of QFT and/or T-SPOT.TB) or TST. Further, studies targeting risk groups (i.e. not population-based) such as healthcare workers, drug users, prison inmates, HIV-positive patients and patients with inflammatory-mediated disease, among others, were excluded to avoid overestimation of LTBI prevalence during targeted screening. Additionally, studies that applied interventions that could affect IGRA and TST results, and studies that selected their population based on specific test results were excluded. In studies of risk groups along with control groups, control subgroups were included if no exclusion criteria were found (e.g. healthy controls). The most comprehensive paper, i.e. largest sample size or most detailed IGRA/TST results, was included when the same data were reported in more than one publication. Authors were contacted for clarification when the methodology was unclear.

We established criteria for assessment of the quality of the included studies, adopted from the Cochrane collaboration for analytical studies [15], on the STROBE (Strengthening the Reporting of Observational Studies in Epidemiology) guidelines for reporting observational studies [16] and according to a global prevalence review [17]. All included studies were assessed for quality on four criteria: quality of sampling method; quality of selection method; response rate; and quality of prevalence assessment. These four criteria were evaluated on a three-point numerical scale $(0,1$ or 2$)$ and involved assessment of internal and external validity and attrition bias (supplementary material section C).

\section{Data extraction}

The following information was extracted: first author, year of publication, study design, study date, study population description, age, exclusion criteria, eligible and invited study population, participants and included participants. Enrolment data were used for the full study enrolment when data for selected subgroups were missing. Furthermore, we extracted measures of TB verification, number of individuals with $\mathrm{TB}$ at the time of screening and whether they were excluded from the final results, sample size, type of test (i.e. version of QFT, T-SPOT.TB and TST) and IGRA- and TST-positive, as well as TST cut-offs. We only used baseline results from studies employing two-step TST to avoid a boosting phenomenon [18]. Indeterminate QFT results were registered, and whether they were excluded or not, and similarly for indeterminate T-SPOT.TB. When IGRA results for more than one cut-off point were presented, we used the manufacturers' instructions for interpretation $[19,20]$. Due to the availability of LTBI survey data, which varied between years and areas, the latest country-specific incidence rates of active TB (including people living with HIV) and country population sizes were gathered in July 2018 from the WHO global TB database [21].

\section{Definition of incidence intervals and evaluation of IGRA and TST results}

In order to enable extrapolation of LTBI prevalence to countries with no data, we divided countries into three intervals of TB incidence: low (0-10 cases per 100000 person-years), intermediate (11-120 cases per 100000 person-years) and high (>120 cases per 100000 person-years). To our knowledge, there is no consensus on defining the upper limit of the intermediate TB incidence interval, and we based our definition on a combination of published data and visual inspection of the latest WHO estimates of TB incidence [3]. To accommodate this uncertainty, we sequentially performed statistical analysis on an upper limit defined as all numbers between 20 and 150 and included the resulting range of final global IGRA and TST estimates. The upper limit of the low interval was based on the WHO framework towards TB elimination in low-incidence countries [22].

We calculated individual prevalence estimates for both IGRA and TST used in all studies and the primary estimate was based on a TST 10-mm cut-off and IGRA tests without considering indeterminate results. 
However, other ways of calculating estimates were considered. In studies including both QFT and T-SPOT.TB, we calculated a sample size weighted mean prevalence and used the QFT sample size as denominator. When both QFT-G, QFT Gold In-Tube (QFT-GIT) and/or QFT Gold Plus (QFT-Plus) results were presented, we prioritised data from the newest test version (i.e. QFT-GIT or QFT-Plus). The reported estimate was based on excluding indeterminate IGRA results, as it cannot be ascertained whether these are truly positive or negative. In order to compare different strategies for calculating IGRA results, we included the following strategies as a sensitivity analysis. 1) Excluding indeterminate results when possible; 2) including indeterminate results in the denominator and regarding them as negative in the numerator; and 3) in a worst-case scenario including indeterminate results in the denominator and regarding them as positive in the numerator. We did not have access to quantitative IGRA data, and thus the proposed grey zone for QFT results $\left(0.20-0.70 \mathrm{IU} \cdot \mathrm{mL}^{-1}\right)$ could not be evaluated [23]. We calculated TST results in three different ways: 1) cut-off at $5 \mathrm{~mm}$ or as close as possible; 2) cut-off at $10 \mathrm{~mm}$ or as close as possible; and 3) cut-off at $15 \mathrm{~mm}$ or as close as possible. Exact 95\% confidence intervals were calculated for all studies and estimates.

\section{Meta-analysis and statistical analysis}

Study prevalence proportions of LTBI based on IGRA and TST were divided into three groups according to the aforementioned TB incidence intervals using WHO TB incidence rates. First, proportions were transformed using the Freeman-Tukey double arcsine method [24]. We assessed a great variation within the study populations, possibly affecting study estimates, and therefore chose to employ a random-effects model, as used in similar studies [25]. In the random effects analysis, increased sample size increases the weight of a study, but the more the study result varies from the other studies in the analysis, the more the weight decreases. This prevents very large studies in one country from affecting the overall result, but provides very small studies with a relatively high weight considering their small population sizes [26]. A pooled inverse variance weighted random effects analysis was performed on each TB incidence group using the DerSimonian and Laird [27] method. Clopper-Pearson 95\% confidence intervals were calculated for each study and for the TB incidence interval pooled estimates. We calculated weights of each TB incidence interval estimate by dividing the pooled country population size of each TB incidence interval with the global population size. Then we calculated a global prevalence of LTBI by weighting the TB incidence interval prevalence estimates according to the population size represented. Study estimate heterogeneity was evaluated using $\mathrm{I}^{2}$ statistics for each incidence interval. Furthermore, we assessed the impact on difference between IGRA and TST global estimates by excluding single-test studies. Using the TB incidence interval estimates for LTBI, we calculated LTBI prevalence estimates for each WHO region by weighting the three incidence interval estimates according to pooled population sizes of the same TB incidence intervals of each WHO region and compared them with WHO estimates. Finally, a Spearman's rank correlation coefficient was calculated to evaluate the relationship between IGRA- and TST-based LTBI prevalence and WHO incidence rates. Statistical analyses were performed using the meta 4.9-2 package in $\mathrm{R}$ (version 3.5.1).

\section{Results}

In total, 8328 search results were identified through MEDLINE ( $\mathrm{n}=2024)$, Embase $(\mathrm{n}=1936)$, Scopus $(n=2394)$, and Web of Science $(n=1974)$. After removal of duplicates, 3280 studies remained, of which 770 full texts were assessed for eligibility, and 682 were excluded. 88 quantitative studies fulfilled the criteria for inclusion (figure 1; studies listed in supplementary material section D) [13, 28-113]. Among 36 countries represented, 41 IGRA $(n=67167)$ and 67 TST $(n=284644)$ estimates were available. Annual TB incidence rates ranged from 0.8 per 100000 in the United Arab Emirates [28] to 781 per 100000 in South Africa [106]. The mean age reported ranged from $~ 51$ months [41] to 82.3 years [66].

Among the included studies, 36 used one or more variants of the QFT assay, including individuals sampled with QFT Gold ( $\mathrm{n}=8262)$ [29, 36, 48, 58, 59, 70, 78, 83, 94], QFT-GIT ( $\mathrm{n}=56327)$ [13, 28, 39, 42, $45,49,51,57,60,67,71,72,79-81,83,86-88,93,96,104-107,110,113]$ and a single study using QFT-Plus ( $n=829)$ [89]. Seven studies used T-SPOT.TB $(n=5547)[31,62,65,72,90,104,114]$ and two of these included simultaneous QFT-GIT [72, 104]. In total, 41 studies used one or more IGRA tools ( $\mathrm{n}=67167$ individuals) $[13,28,29,31,36,39,42,45,48,49,51,57-60,62,65,67,70-72,78-81,83$, 86-90, 93, 94, 96, 104-107, 110, 113, 114]. 67 studies had TST results ( $\mathrm{n}=284644$ individuals) [13, 30-38, $40-47,49-56,61,63,65,66,68,69,72-77,80,82-87,91-93,95-109,112,113,115] .20$ studies used both IGRA and TST $[13,31,36,42,45,49,51,65,72,80,83,86,87,93,96,104-107,113]$. The pooled sample sizes of studies using TST were larger than studies using IGRA in all intervals and largest in the high-incidence interval (table 1).

The studies included country estimates $(n=36)$ covering all incidence intervals and WHO regions (supplementary material section E). A world map was compiled showing all countries with original LTBI prevalence data coloured in darker variants of blue, orange and red, depending on which of the three TB 


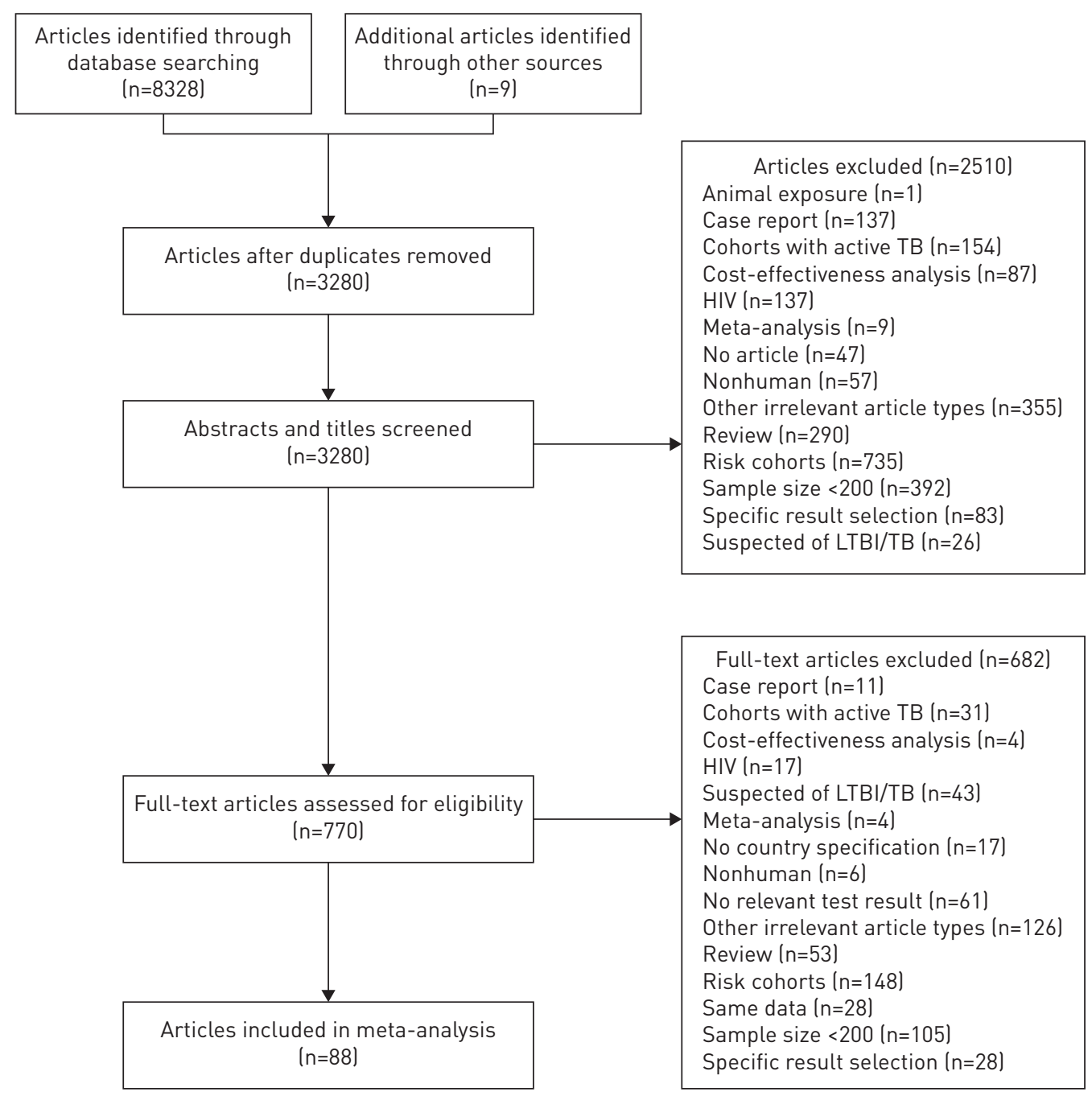

FIGURE 1 Study selection. Flow chart of study inclusion. Studies are listed in supplementary material section D. TB: tuberculosis; LTBI: latent TB infection.

incidence intervals they were within (i.e. low, intermediate or high) (figure 2). For the remaining countries without any current data $(n=159)$, we used the weighted LTBI prevalence estimate of their respective TB incidence interval, and coloured the countries in a lighter version of aforementioned colours.

The global prevalence of LTBI was 24.8\% (95\% CI 19.7-29.9\%) and 21.2\% (95\% CI 17.9-24.4\%) according to IGRA and TST $(10 \mathrm{~mm})$ results, respectively. Prevalence of LTBI by TB incidence intervals for TSTs and IGRAs are shown in the forest plots in figures 3 and 4. There was a strong monotonic relationship between WHO TB incidence rates and LTBI prevalence based on both IGRAs $\left(r_{\mathrm{s}}=0.706, \mathrm{p}<0.0001\right)$ and TSTs $\left(r_{\mathrm{s}}=0.697, \mathrm{p}<0.0001\right)$. The between-study estimate heterogenicity of the low, intermediate and high

\section{TABLE 1 Pooled sample sizes of included studies}

IGRA sample size

TST sample size

Total sample size

$\begin{array}{ll}\text { TB incidence intervals } & \\ \quad \text { Low } & 16628(24.8) \\ \text { Intermediate } & 37392(55.7) \\ \text { High } & 13147(19.6) \\ \text { Total sample size } & 67167(19.1)\end{array}$

$104379(36.7)$
$63432(22.3)$
$116833(41.0)$
$284644(80.9)$

$121007(34.4)$

$100824(28.7)$

$129980(36.9)$

$351811(100.0)$

Data are presented as $\mathrm{n}(\%)$. Pooled sample sizes by interferon- $\gamma$ release assay (IGRA), tuberculin skin test (TST) (10-mm cut-off) and in total. Categorisation of countries in tuberculosis (TB) incidence intervals is listed in supplementary material section $\mathrm{E}$. 


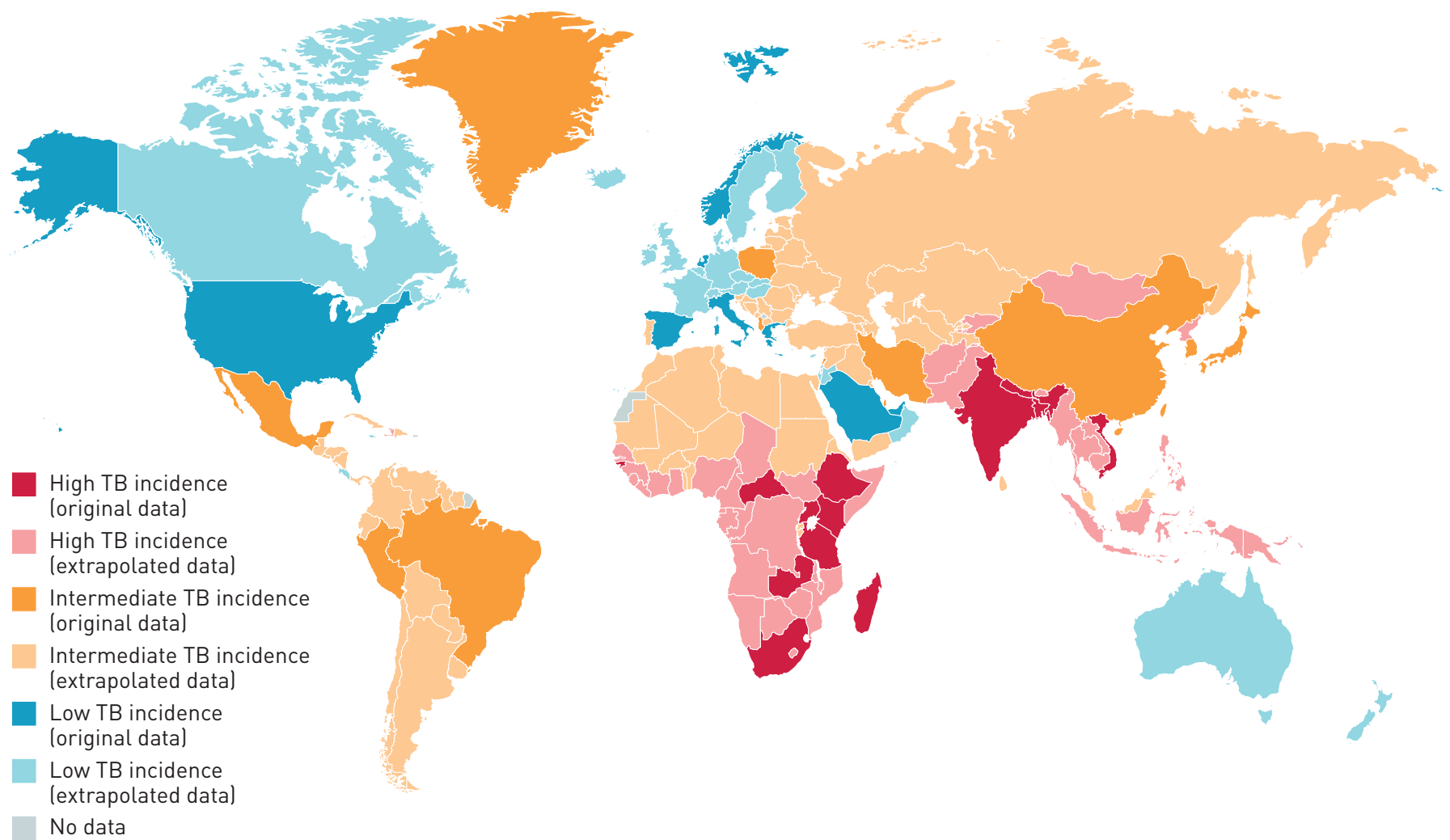

FIGURE 2 World map of countries by tuberculosis (TB) incidence. High, intermediate and low active TB incidence countries are shown, corresponding to average latent TB infection (LTBI) prevalence of $28-36 \%, 19-20 \%$ and $3-5 \%$, respectively (from figures 3 and 4 ). Darker shades of the colours indicate areas with original LTBI prevalence data, lighter shaded colours indicate countries where the weighted estimate of the country's TB incidence interval has been used.

TB incidence interval, calculated with $\mathrm{I}^{2}$ statistics, was $97 \%, 99 \%$ and $99 \%$, respectively, for IGRA and $100 \%$ for all three incidence intervals with TST.

Including indeterminate results in the denominator, and regarding indeterminate results as negative, global prevalence based on IGRA was $24.2 \%$ (95\% CI 19.2-29.2\%). In a worst-case scenario, regarding indeterminate results as positive, global prevalence was estimated to be $26.3 \%$ (95\% CI $21.0-31.6 \%$ ). Based on TST results, we calculated a global prevalence of $24.1 \%$ (95\% CI $20.2-28.0 \%$ ), $21.2 \%$ (95\% CI $17.9-$ $24.4 \%$ ) and $17.4 \%$ (95\% CI 14.4-20.4\%) using 5-, 10- and 15-mm cut-offs, respectively. If only considering studies that used IGRA and TST tests concurrently, 20 studies remained, with a pooled population of 43861 (IGRA) and 44238 (TST) [13, 31, 36, 45, 49, 51, 65, 72, 80, 83, 86, 87, 93, 96, 104-107, 113]. The global IGRA estimate was then $25.2 \%$ (95\% CI 19.8-30.7\%) (indeterminate results excluded) and the global TST estimate $(10 \mathrm{~mm})$ was $27.1 \%$ (95\% CI $18.9-35.3 \%)$. When calculations were performed sequentially with the upper limit of the intermediate $\mathrm{TB}$ prevalence interval defined as all numbers between 20 and 150, the global estimate ranged between 22.6-25.0\% (IGRA) and 20.6-22.3\% (TST).

These new estimates based on both IGRA and TST (10-mm cut-off) were lower than WHO estimates in all WHO regions (table 2). Most notably, new TST estimates of Southeast Asia and Western Pacific were more than one-third lower than the 1999 estimates. IGRA estimates were slightly higher in all WHO regions compared with new TST estimates.

The quality of the studies included varied ranging from 0 to 8 points out of a possible 8 (references available in supplementary material section C). Most studies employed convenience sampling ( $\mathrm{n}=60$ out of 88). Information on response rate was presented in $42(47.7 \%)$ out of 88 studies. Indeterminate results were reported in $30(73.2 \%)$ out of 41 studies on IGRA, of which four had indeterminate results constituting $>5 \%$ of all results $[31,60,65,96] .27(40.3 \%)$ out of 67 studies reported a 15 -mm TST cut-off, $56(83.6 \%)$ a $10-\mathrm{mm}$ cut-off and $26(38.8 \%)$ a 5 -mm cut-off.

\section{Discussion}

In this study, we present an update of the global LTBI prevalence estimate, for the first time based directly on both IGRA and TST results, the tests currently being used to diagnose and select LTBI subjects eligible 


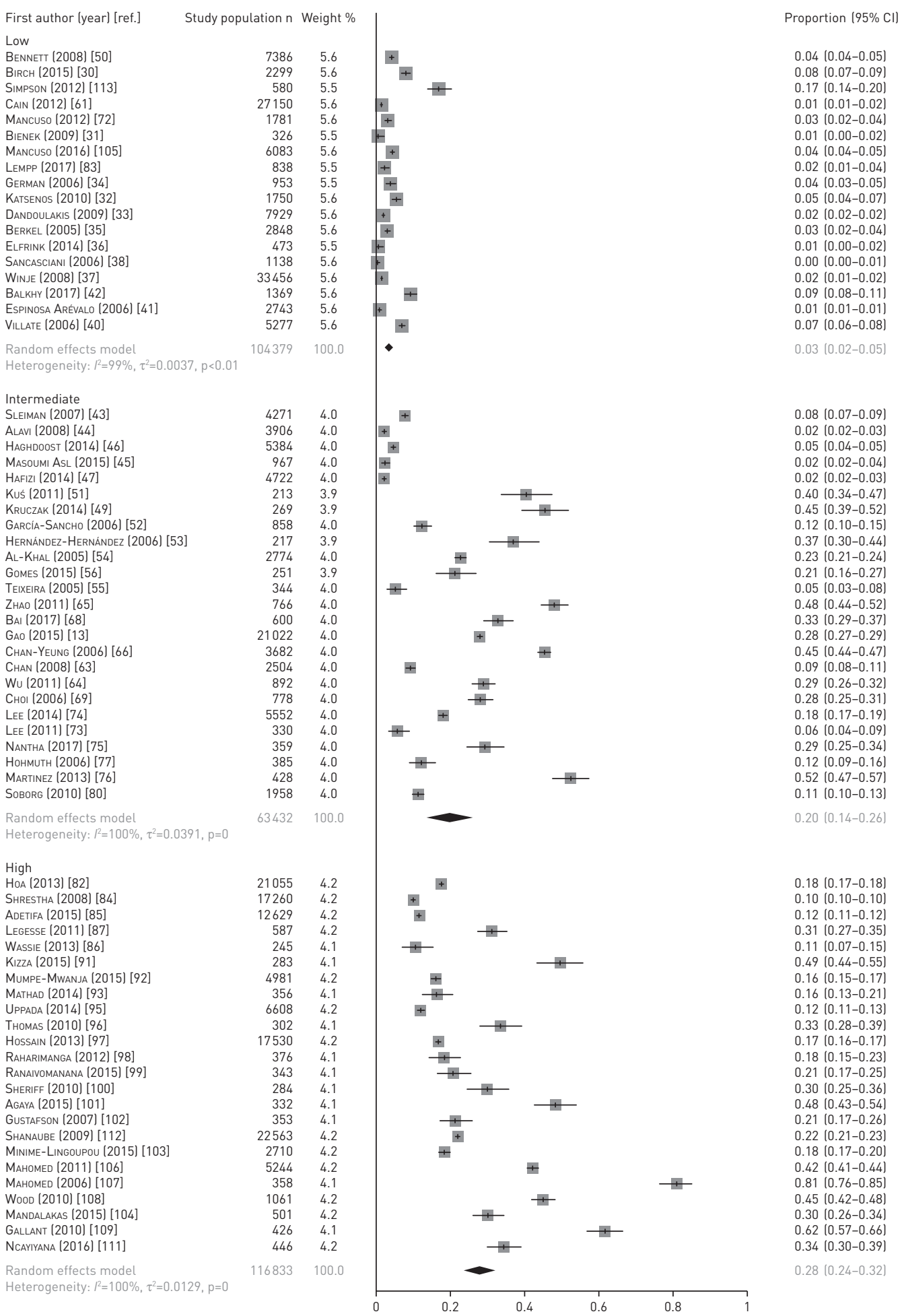

FIGURE 3 Forest plot of tuberculin skin test (TST) data. Prevalence of latent tuberculosis infection by tuberculosis incidence intervals using random effects model. Latent infection based on TST with a 10-mm cut-off.

for preventive therapy. Data were gathered from $>350000$ IGRA and TST results covering all WHO regions. New IGRA and TST estimates were comparable in most regions, but systematically slightly higher for IGRAs. Our global estimate of the LTBI prevalence is an update of the WHO estimate from 1999 and very much in line with a similar annual risk of infection-based modelling study from 2016 suggesting a global prevalence of LTBI at 23\% [2]. Our findings support that the global prevalence of LTBI is no longer 


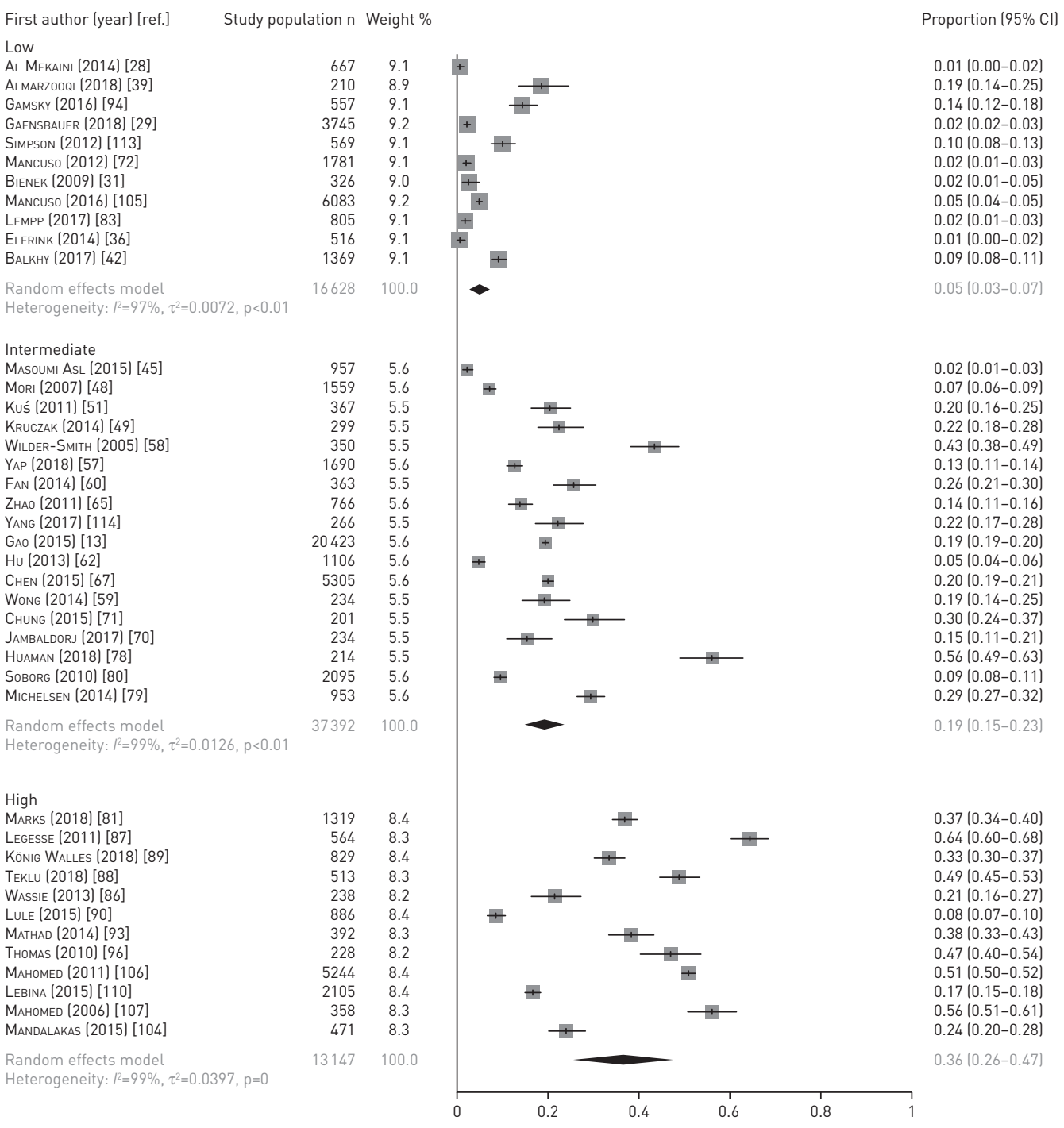

FIGURE 4 Forest plot of interferon- $\gamma$ release assay (IGRA) data. Prevalence of latent tuberculosis infection by tuberculosis incidence intervals using random effects model. Latent infection based on IGRA with indeterminate excluded.

a third of the world population, but closer to one-fourth, with large regional differences, which in this test-based study is similar to the old and new modelled estimates; the reductions are in many aspects, although not completely, aligned with the new prevalences modelled by HouBEN and DodD [2].

In accordance with the previous WHO estimates from 1999 [1] as well as with the new estimates by Houben and Dodd [2], we found that Southeast Asia is the region with the highest LTBI prevalence. In contrast to both previous estimates, we observed that Africa had the second highest prevalence with $26.6 \%$ (IGRA) and 33.6\% (TST) whereas Houben and Dodp [2] reported a regional prevalence of only 22\%, considerably lower than the previous WHO estimate of 35\%. According to WHO, the TB incidence rates in Africa have been decreasing since 2005 [3]. However, before 2005, while end-targets for TB fell in other regions, Africa saw a rise since monitoring began in the early 1990s. This could partly explain the slower pace in reducing LTBI prevalence in Africa, as is indicated by our estimates. Sub-Saharan Africa in particular is a high-endemic region, and active TB is prioritised due to the high burden of disease and limited resources while LTBI is mainly a concern for close contacts to smear positive TB patients and people living with HIV [116]. Of further interest, we found considerably lower estimates for the Western Pacific region with 20.7\% (IGRA) and 20.3\% (TST) whereas HoubEN and DodD [2] report 27.9\%, closer to the previous WHO estimate of $36 \%$. Our estimates are more in line with specific estimates for China of $19 \%$ [13], which makes the estimate plausible since China constitutes $73 \%$ of the Western Pacific region population. 
TABLE 2 New and old estimates of latent tuberculosis infection (LTBI) prevalence by World Health Organization (WHO) region

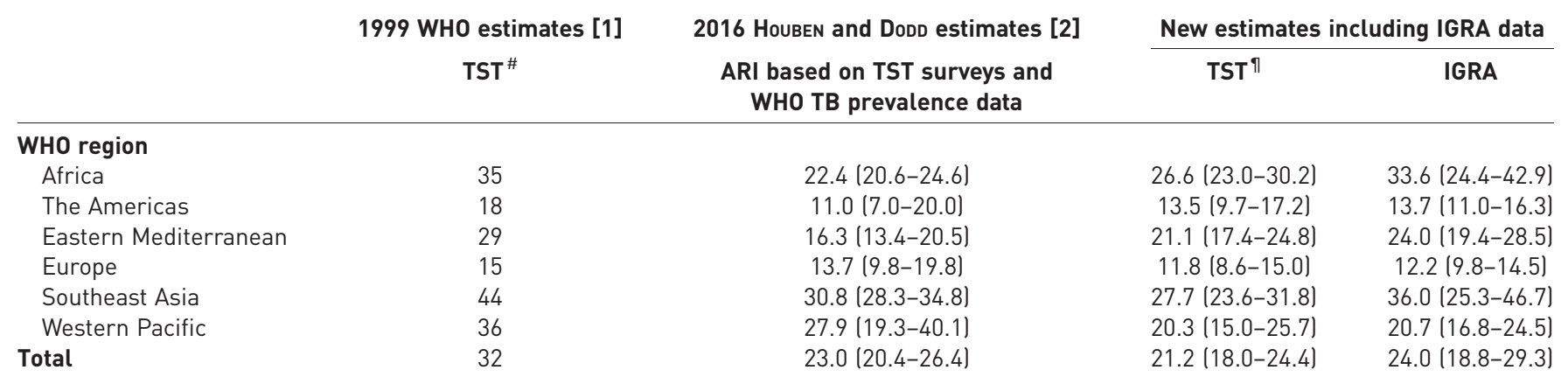

Data are presented as \% or \% (95\% CI). Global and regional prevalences of LTBI displaying 1999 WHO estimates [1], new modelling estimates from 2016 by HouBEN and DoDD [2] and our current estimates, including interferon- $\gamma$ release assay (IGRA) survey data. TST: tuberculin skin test; ARI: annual risk of infection; TB: tuberculosis. " : partly based on annual risk of infection and Styblo's rule; " : 10-mm cut-off for TST.

Estimating the true rate of LTBI is highly challenging due to the absence of a gold standard for LTBI. As both IGRA and TST detect memory T-cell response to previous $M$. tuberculosis antigen exposure, a positive test is not necessarily associated with infection of viable bacteria [117]. Of note, the tests are insufficient in detecting progression into active TB with very low positive predictive values of $2.7 \%(95 \%$ CI $2.3-3.2 \%$ ) for IGRAs and 1.5\% (95\% CI 1.2-1.7\%) for TSTs in one systematic review [118]. Recently, a new version of IGRA, QFT-Plus, has been introduced containing additional TB antigens stimulating both CD4 and CD8 T-cells [119], which according to the manufacturer could result in an enhanced sensitivity; however, so far there is a high agreement (>95\%) between the QFT-Plus and older versions of the QFT $[120,121]$.

IGRA and TST are currently used to diagnose candidates for preventive LTBI therapy, and consequently, we applied these as surrogate markers for ongoing TB exposure [122]. We chose to present the estimate based on the TST $10-\mathrm{mm}$ cut-offs and based on exclusion of indeterminate IGRA results from the numerator and denominator as a compromise between sensitivity and specificity. The variability for using other strategies was low. It was unexpected that despite a higher specificity of the blood test, almost all LTBI estimates using IGRA were higher than the senescent skin test. However, this may be dependent on the cut-off applied, which is not clearly established for any of the tests with regards to LTBI. We speculate that one reason for the systematically higher IGRA estimates, compared to TST, may be that IGRAs are slightly more sensitive to detect LTBI than TST when using the 10 -mm cut-off. When a 5-mm cut-off was applied, the tests were more comparable at $24.8 \%$ and $24.1 \%$ for IGRA and TST respectively. Furthermore, the tests suffer from variability, and the most optimal cut-off levels are under discussion, in particular for the IGRAs where a grey zone for QFT in the range of $0.20-0.70 \mathrm{IU} \cdot \mathrm{mL}^{-1}$ has been suggested $[23,123]$. We chose to use the established cut-offs suggested by manufacturers and international guidelines as quantitative IGRA results were very rarely available. However, false-positive QFT results in the grey zone do exist and may have overestimated IGRA results marginally [23, 124]. Another possible explanation could be a baseline difference in sampling, and we did find a slightly higher estimate from TST when limiting results to studies with both tests. This finding may indicate that IGRAs are not only more specific, but also more sensitive, although this is difficult to assess in the lack of a gold-standard test for LTBI. Even though it has previously been perceived that TST is more frequently positive, as it also captures BCG vaccination and other mycobacteria, we may actually here see a display of the fact that in populations where BCG is given at infancy, it has limited impact on the test result [125]. Impaired immunity such as HIV may have higher impact on TST results than IGRAs.

Our study has several limitations. First, crude estimates were based on small study population sizes, especially for IGRAs, in studies of varying sampling technique and quality. We performed vast extrapolation with several assumptions; most notably that our pooled estimates of each incidence interval represented the mean prevalence among the large populations represented by each interval. Second, the exclusion of patients with inflammatory disease was based on the assumption that this group may show inferior sensitivity to IGRA and TST due to the underlying disease and/or concurrent immunosuppressive therapy. Third, age was assumed to be representative of the global age prevalence in the study populations included, and was not accounted for in our extrapolation. Age could act as a surrogate marker for accumulated TB exposure and thus be a risk factor as several studies indicate $[13,108]$. As outlined in 
supplementary material section D, the LTBI prevalence data illustrate a consistent effect of age when comparing populations of younger and older surveyed study participants (i.e. South Africa 16-56\%, Mexico $12-36.9 \%$, Singapore $12.6-43.4 \%$, Spain $0.9-9.3 \%$ and USA $1.5-8 \%$ ), but that does not necessarily imply that the studies are not representative. In a meta-analysis based on published prevalence surveys, it is not possible to adjust reliably for age, and we acknowledge that this may introduce bias. Yet we have no indications that the surveyed populations are skewed towards being particularly young or old individuals, which would force the prevalence estimate up or down. Moreover, children and adults were represented in all incidence intervals. Our estimate has the strength of being based on individual measurements in populations across a large number of countries across the world instead of being based on mathematical models. Fourth, the relatively wide confidence intervals around specific prevalence estimates, which makes monitoring of incremental statistical changes in LTBI prevalence difficult, is another limitation. This is a reflection of the data available with low sample sizes in some of the surveys and large total populations in the surveyed countries, including populations with extrapolated prevalences. This variability of study estimates due to large and diverse populations is also reflected in the high heterogeneity $(\geqslant 97 \%)$ calculated with $\mathrm{I}^{2}$ statistics. We believe that although this represents uncertainty in our estimates, all studies not excluded represent important parts of the total background population, which is undoubtedly diverse, yet an indication that the prevalences should be interpreted with caution. Fifth, the included studies spanned 15 years and we assumed no development in TB prevalence during this period of years. Although these parameters were accounted for in the modelling study by Houben and DodD [2], we found remarkably comparable estimates. Finally, we have excluded data on high-risk populations, and their contribution to the global burden of LTBI may therefore not be sufficiently represented. Studies with a focus on migrant populations were excluded, and migrants may not have been well represented in population surveys, which may also have led to an underestimation of the LTBI prevalence; in low-incidence countries, they may constitute the majority of LTBI cases, e.g. in Australia, it is estimated that only $6.8 \%$ of all those with LTBI are Australian-born [126].

As always in meta-analyses, the selection of studies may lead to bias, and extrapolations to countries with no data will most certainly introduce bias. Yet, it is important to keep in mind that models and estimations are also not free of bias, in particular if based on data of active TB and assumptions on infectiousness, which we hold is more uncertain than published survey data on IGRA/TST results. Furthermore, models using the Styblo rule will face difficulties in taking the age factor into consideration in case of changing epidemiology and population structures; hence, modelling may not lead to a better estimate, if the estimate is based on assumptions that are difficult to adequately predict. For instance, children are less likely to transmit than adults but perhaps that is controlled for, as they are less likely to be smear-positive. However, this depends on the age and may not apply to children aged $>15$ years. With no golden standard for diagnosing LTBI, and no method available to measure viable $M$. tuberculosis and to distinguish between "true" LTBI and cleared infection, we believe that assessment of global prevalences of LTBI must be based on the measurements currently available and those used in clinical practice. The decision to initiate preventive therapy will not be based on assumptions but on individual testing, and although age is important in the assessment of the probability of infection, in the end, it will be the test outcome that determines who is eligible for therapy and who is not.

In conclusion, we estimate one-fourth of the world's population to be latently infected with TB, in the first study applying both IGRA and TST surveys. LTBI still represents an enormous reservoir of potential reactivated TB and this must be recognised as a considerable obstacle, and as a point of intervention, in reaching the End TB Strategy goals of 2050 .

Author contributions: C. Wejse and A. Cohen conceived and designed the study. All data were collected by A. Cohen and V.D. Mathiasen. A. Cohen and V.D. Mathiasen had access to all obtained data and conducted statistical analyses. A. Cohen drafted the first manuscript with contributions from V.D. Mathiasen, T. Schön and C. Wejse. All authors interpreted data as well as contributed with intellectual content to the final manuscript. C. Wejse and T. Schön were study supervisors. All authors agree with the results and conclusions of this article.

Conflict of interest: None declared.

Support statement: This study was supported by NovoNordisk Foundation (NNF15OC0018034; C. Wejse), the Swedish Research Council (201602043; T. Schön) and the Swedish Heart and Lung Foundation (20150236; T. Schön). Funding information for this article has been deposited with the Crossref Funder Registry.

\section{References}

1 Dye C, Scheele S, Dolin P, et al. Consensus statement. Global burden of tuberculosis: estimated incidence, prevalence, and mortality by country. WHO Global Surveillance and Monitoring Project. JAMA 1999; 282: 677-686.

2 Houben RMGJ, Dodd PJ. The global burden of latent tuberculosis infection: a re-estimation using mathematical modelling. PLoS Med 2016; 13: e1002152. 
World Health Organization. Global Tuberculosis Report 2018. www.who.int/tb/publications/global_report/en/ Date last accessed: February 28, 2019. Date last updated: February 28, 2019.

4 Wejse C. Tuberculosis elimination in the post Millennium Development Goals era. Int J Infect Dis 2015; 32: 152-155.

5 Churchyard GJ, Swindells S. Controlling latent TB tuberculosis infection in high-burden countries: a neglected strategy to end TB. PLoS Med 2019; 16: e1002787.

6 Esmail H, Barry CE, Young DB, et al. The ongoing challenge of latent tuberculosis. Philos Trans R Soc B Biol Sci 2014; 369: 20130437.

7 Styblo K. The relationship between the risk of tuberculous infection and the risk of developing infectious tuberculosis. Bull Int Union Tuberc Lung Dis 1985; 60: 117-119.

8 van Leth F, van der Werf MJ, Borgdorff MW. Prevalence of tuberculous infection and incidence of tuberculosis: a re-assessment of the Styblo rule. Bull World Health Organ 2008; 86: 20-26.

9 Virenfeldt J, Rudolf F, Camara C, et al. Treatment delay affects clinical severity of tuberculosis: a longitudinal cohort study. BMJ Open 2014; 4: e004818.

10 Petersen E, Khamis F, Migliori GB, et al. De-isolation of patients with pulmonary tuberculosis after start of treatment - clear, unequivocal guidelines are missing. Int J Infect Dis 2017; 56: 34-38.

11 Getahun H, Matteelli A, Chaisson RE, et al. Latent Mycobacterium tuberculosis infection. N Engl J Med 2015; 372: 2127-2135.

12 Pai M, Zwerling A, Menzies D. Systematic review: T-cell-based assays for the diagnosis of latent tuberculosis infection: an update. Ann Intern Med 2008; 149: 177-184.

13 Gao L, Lu W, Bai L, et al. Latent tuberculosis infection in rural China: baseline results of a population-based, multicentre, prospective cohort study. Lancet Infect Dis 2015; 15: 310-319.

14 Liberati A, Altman DG, Tetzlaff J, et al. The PRISMA statement for reporting systematic reviews and meta-analyses of studies that evaluate healthcare interventions: explanation and elaboration. J Clin Epidemiol 2009; 62: e1-e34.

15 Higgins JPT, Altman DG, Gotzsche PC, et al. The Cochrane Collaboration's tool for assessing risk of bias in randomised trials. BMJ 2011; 343: d5928.

16 von Elm E, Altman DG, Egger M, et al. Strengthening the Reporting of Observational Studies in Epidemiology (STROBE) statement: guidelines for reporting observational studies. BMJ 2007; 335: 806-808.

17 Romani L, Steer AC, Whitfeld MJ, et al. Prevalence of scabies and impetigo worldwide: a systematic review. Lancet Infect Dis 2015; 15: 960-967.

18 Murthy M, Selvam S, Jesuraj N, et al. Two-step tuberculin skin testing in school-going adolescents with initial 04 millimeter responses in a high tuberculosis prevalence setting in south India. PLoS One 2013; 8: e71470.

19 QuantiFERON-TB Gold (QFT) ELISA Package Insert - UK. www.quantiferon.com/wp-content/uploads/2017/04/ English_QFT_ELISA_R04_082016.pdf Date last accessed: February 28, 2019. Date last updated: August, 2016. T-SPOT.TB Package Insert. PI-TB-US-V4. www.tspot.com/wp-content/uploads/2012/01/PI-TB-US-v4.pdf Date last accessed: February 28, 2019. Date last updated: March, 2013.

21 World Health Organization (WHO). WHO's Global Tuberculosis Database. www.who.int/tb/data/en/ Date last accessed: February 28, 2019.

22 World Health Organization (WHO). Framework Towards Tuberculosis Elimination in Low-incidence Countries. 2014. www.who.int/tb/publications/elimination_framework/en/ Date last accessed: February 28, 2019.

23 Nemes E, Rozot V, Geldenhuys H, et al. Optimization and interpretation of serial QuantiFERON testing to measure acquisition of Mycobacterium tuberculosis infection. Am J Respir Crit Care Med 2017; 196: 638-648. Miller JJ. The inverse of the Freeman-Tukey double arcsine transformation. Am Stat 1978; 32: 138-138.

25 Apriani L, McAllister S, Sharples K, et al. Latent tuberculosis infection in healthcare workers in low- and middle-income countries: an updated systematic review. Eur Respir J 2019; 53: 1801789.

26 Hill NR, Fatoba ST, Oke JL, et al. Global prevalence of chronic kidney disease - a systematic review and meta-analysis. PLoS One 2016; 11: e0158765.

27 DerSimonian R, Laird N. Meta-analysis in clinical trials. Control Clin Trials 1986; 7: 177-188.

28 Al Mekaini LA, Al Jabri ON, Narchi H, et al. The use of an interferon-gamma release assay to screen for pediatric latent tuberculosis infection in the eastern region of the Emirate of Abu Dhabi. Int J Infect Dis 2014; 23: 4-7.

29 Gaensbauer J, Gonzales B, Belknap R, et al. Interferon-gamma release assay-based screening for pediatric latent tuberculosis infection in an urban primary care network. J Pediatr 2018; 200: 202-209.

30 Birch SJ, Golbeck AL. The effectiveness of screening with interferon-gamma release assays in a university health care setting with a diverse global population. J Am Coll Heal 2015; 63: 180-185.

31 Bienek DR, Chang CK. Evaluation of an interferon-gamma release assay, T-SPOT.TB, in a population with a low prevalence of tuberculosis. Int J Tuberc Lung Dis 2009; 13: 1416-1421.

32 Katsenos S, Nikolopoulou M, Konstantinidis AK, et al. Interferon-gamma release assay clarifies the effect of bacille Calmette-Guérin vaccination in Greek army recruits. Int J Tuberc Lung Dis 2010; 14: 545-550.

33 Dandoulakis M, Roussos N, Karageorgopoulos DE, et al. Trends of tuberculin skin test positivity rate among schoolchildren in Attica, Greece. Scand J Infect Dis 2009; 41: 195-200.

34 German V, Giannakos G, Kopterides P, et al. Prevalence and predictors of tuberculin skin positivity in Hellenic Army recruits. BMC Infect Dis 2006; 6: 102

35 Berkel GM, Cobelens FG, de Vries G, et al. Tuberculin skin test: estimation of positive and negative predictive values from routine data. Int J Tuberc Lung Dis 2005; 9: 310-316.

36 Elfrink F, van den Hoek A, Mensen ME, et al. Screening travellers to high-endemic countries for infection with Mycobacterium tuberculosis using interferon gamma release assay; a prospective study. BMC Infect Dis 2014; 14: 515.

37 Winje BA, Oftung F, Korsvold GE, et al. School based screening for tuberculosis infection in Norway: comparison of positive tuberculin skin test with interferon-gamma release assay. BMC Infect Dis 2008; 8: 140.

38 Sancasciani S, Santori D, Bellugi A, et al. Prevalence of tuberculosis infection among teen-agers in Tuscany. J Prev Med Hyg 2006; 47: 151-154. 
39 Almarzooqi F, Alkhemeiri A, Aljaberi A, et al. Prospective cross-sectional study of tuberculosis screening in United Arab Emirates. Int J Infect Dis 2018; 70: 81-85.

40 Villate JI, Ibáñez B, Cabriada V, et al. Analysis of latent tuberculosis and Mycobacterium avium infection data using mixture models. BMC Public Health 2006; 6: 240.

41 Espinosa Arévalo M, Vázquez Gallardo R, Gayoso Diz P. La prueba de tuberculina en los controles del niño sano. ¿Debemos cambiar nuestra práctica? [The tuberculin skin test in a healthy child program: should we change our practice?] An Pediatr 2006; 65: 225-228.

42 Balkhy HH, El Beltagy K, El-Saed A, et al. Prevalence of latent Mycobacterium tuberculosis infection (LTBI) in Saudi Arabia; population based survey. Int J Infect Dis 2017; 60: 11-16.

43 Sleiman R, Al-Tannir M, Dakdouki G, et al. Interpretation of the tuberculin skin test in bacille Calmette-Guérin vaccinated and nonvaccinated school children. Pediatr Infect Dis J 2007; 26: 134-138.

44 Alavi SM, Sefidgaran GH. Tuberculin survey among school-aged children in Ahvaz, Iran, 2006. Int J Infect Dis 2008; 12: 406-409.

45 Masoumi Asl H, Alborzi A, Pourabbas B, et al. QuantiFERON-TB Gold and Tuberculin Skin test for the diagnosis of latent tuberculosis infection in children. Iran J Med Sci 2015; 40: 411-417.

46 Haghdoost AA, Afshari M, Baneshi MR, et al. Estimating the annual risk of tuberculosis infection and disease in southeast of Iran using the Bayesian mixture method. Iran Red Crescent Med J 2014; 16: e15308.

47 Hafizi H, Aliko A, Sharra E, et al. Results of a tuberculin skin testing survey in Albania. J Infect Dev Ctries 2014; 8: 310-314.

48 Mori $\mathrm{T}$, Harada $\mathrm{N}$, Higuchi $\mathrm{K}$, et al. Waning of the specific interferon-gamma response after years of tuberculosis infection. Int J Tuberc Lung Dis 2007; 11: 1021-1025.

49 Kruczak K, Duplaga M, Sanak M, et al. Comparison of IGRA tests and TST in the diagnosis of latent tuberculosis infection and predicting tuberculosis in risk groups in Krakow, Poland. Scand J Infect Dis 2014; 46: 649-655.

50 Bennett DE, Courval JM, Onorato I, et al. Prevalence of tuberculosis infection in the United States population. Am J Respir Crit Care Med 2008; 177: 348-355.

51 Kuś J, Demkow U, Lewandowska K, et al. Ocena częstości występowania zakażenia prątkiem gruźlicyw populacji województwa mazowieckiego na podstawie wyniku testumierzącego uwalnianie interferonu gamma po stymulacji swoistymi antygenami ESAT-6 i CFP-10. [Prevalence of latent infection with Mycobacterium tuberculosis in Mazovia Region using interferon gamma release assay after stimulation with specific antigens ESAT-6 and CFP-10.] Pneumonol Alergol Pol 2011; 79: 407-418.

52 García-Sancho F MC, García-García L, Jiménez-Corona ME, et al. Is tuberculin skin testing useful to diagnose latent tuberculosis in BCG-vaccinated children? Int J Epidemiol 2006; 35: 1447-1454.

53 Hernández-Hernández E, Alberú J, González-Michaca L, et al. Screening for tuberculosis in the study of the living renal donor in a developing country. Transplantation 2006; 81: 290-292.

54 Al-Khal AL, Bener A, Enarson DA. Tuberculosis among garment workers in an Arabian developing country: State of Qatar. Arch Environ Occup Health 2005; 60: 295-298.

55 Teixeira EG, Menzies D, Comstock GW, et al. Latent tuberculosis infection among undergraduate medical students in Rio de Janeiro State, Brazil. Int J Tuberc Lung Dis 2005; 9: 841-847.

56 Gomes CMF, Terreri MT, Moraes-Pinto Md, et al. Incidence of active mycobacterial infections in Brazilian patients with chronic inflammatory arthritis and negative evaluation for latent tuberculosis infection at baseline a longitudinal analysis after using TNF $\alpha$ blockers. Mem Inst Oswaldo Cruz 2015; 110: 921-928.

57 Yap P, Tan KHX, Lim WY, et al. Prevalence of and risk factors associated with latent tuberculosis in Singapore: a cross-sectional survey. Int J Infect Dis 2018; 72: 55-62.

58 Wilder-Smith A, Foo W, Earnest A, et al. High risk of Mycobacterium tuberculosis infection during the Hajj pilgrimage. Trop Med Int Health 2005; 10: 336-339.

59 Wong SH, Ip M, Tang W, et al. Performance of interferon-gamma release assay for tuberculosis screening in inflammatory bowel disease patients. Inflamm Bowel Dis 2014; 20: 2067-2072.

60 Fan W-C, Ting W-Y, Lee M-C, et al. Latent TB infection in newly diagnosed lung cancer patients - a multicenter prospective observational study. Lung Cancer 2014; 85: 472-478.

61 Cain KP, Garman KN, Laserson KF, et al. Moving toward tuberculosis elimination: implementation of statewide targeted tuberculin testing in Tennessee. Am J Respir Crit Care Med 2012; 186: 273-279.

$62 \mathrm{Hu} \mathrm{Y,} \mathrm{Zhao} \mathrm{Q,} \mathrm{Wu} \mathrm{L,} \mathrm{et} \mathrm{al.} \mathrm{Prevalence} \mathrm{of} \mathrm{latent} \mathrm{tuberculosis} \mathrm{infection} \mathrm{and} \mathrm{its} \mathrm{risk} \mathrm{factors} \mathrm{in} \mathrm{schoolchildren} \mathrm{and}$ adolescents in Shanghai, China. Eur J Public Health 2013; 23: 1064-1069.

63 Chan PC, Chang LY, Wu YC, et al. Age-specific cut-offs for the tuberculin skin test to detect latent tuberculosis in BCG-vaccinated children. Int J Tuberc Lung Dis 2008; 12: 1401-1406.

$64 \mathrm{Wu} \mathrm{X}$, Hou Y, Liang Y, et al. Evaluation of a tuberculosis whole-blood interferon- $\gamma$ chemiluminescent immunoassay among Chinese military recruits. Mol Diagn Ther 2011; 15: 341-346.

65 Zhao J, Wang Y, Wang H, et al. Low agreement between the T-SPOT ${ }^{\varpi}$.TB assay and the tuberculin skin test among college students in China. Int J Tuberc Lung Dis 2011; 15: 134-136.

66 Chan-Yeung M, Cheung AHK, Dai DLK, et al. Prevalence and determinants of positive tuberculin reactions of residents in old age homes in Hong Kong. Int J Tuberc Lung Dis 2006; 10: 892-898.

67 Chen C, Zhu T, Wang Z, et al. High latent TB infection rate and associated risk factors in the eastern China of low TB incidence. PLoS One 2015; 10: e0141511.

68 Bai X, Liang Y, Yang Y, et al. A new method of screening for latent tuberculosis infection: results from army recruits in Beijing in 2014. Immunol Lett 2017; 186: 28-32.

69 Choi CM, Kang CI, Kim DH, et al. The role of TST in the diagnosis of latent tuberculosis infection among military personnel in South Korea. Int J Tuberc Lung Dis 2006; 10: 1342-1346.

70 Jambaldorj E, Han M, Jeong JC, et al. Poor predictability of QuantiFERON-TB assay in recipients and donors for tuberculosis development after kidney transplantation in an intermediate-TB-burden country. BMC Nephrol 2017; $18: 88$.

71 Chung W, Lee K, Jung Y, et al. Serum CXCR3 ligands as biomarkers for the diagnosis and treatment monitoring of tuberculosis. Int J Tuberc Lung Dis 2015; 19: 1476-1484.

72 Mancuso JD, Mazurek GH, Tribble D, et al. Discordance among commercially available diagnostics for latent tuberculosis infection. Am J Respir Crit Care Med 2012; 185: 427-434. 
73 Lee H, Cho SN, Kim HJ, et al. Evaluation of cell-mediated immune responses to two BCG vaccination regimes in young children in South Korea. Vaccine 2011; 29: 6564-6571.

74 Lee SW, Oh SY, Lee JB, et al. Tuberculin skin test distribution following a change in BCG vaccination policy. PLoS One 2014; 9: e86419.

75 Nantha YS, Puri A, Mohamad Ali SZ, et al. Epidemiology of latent tuberculosis infection among patients with and without diabetes mellitus. Fam Pract 2017; 34: 532-538.

76 Martinez L, Arman A, Haveman N, et al. Changes in tuberculin skin test positivity over 20 years in periurban shantytowns in Lima, Peru. Am J Trop Med Hyg 2013; 89: 507-515.

77 Hohmuth BA, Yamanija JC, Dayal AS, et al. Latent tuberculosis infection: risks to health care students at a hospital in Lima, Peru. Int J Tuberc Lung Dis 2006; 10: 1146-1151.

78 Huaman MA, Ticona E, Miranda G, et al. The relationship between latent tuberculosis infection and acute myocardial infarction. Clin Infect Dis 2018; 66: 886-892.

79 Michelsen SW, Soborg B, Koch A, et al. The effectiveness of BCG vaccination in preventing Mycobacterium tuberculosis infection and disease in Greenland. Thorax 2014; 69: 851-856.

80 Soborg B, Koch A, Thomsen VØ, et al. Ongoing tuberculosis transmission to children in Greenland. Eur Respir J 2010; 36: 878-884.

81 Marks GB, Nhung NV, Nguyen TA, et al. Prevalence of latent tuberculous infection among adults in the general population of Ca Mau, Viet Nam. Int J Tuberc Lung Dis 2018; 22: 246-251.

82 Hoa NB, Cobelens FGJ, Sy DN, et al. First national tuberculin survey in Viet Nam: characteristics and association with tuberculosis prevalence. Int J Tuberc Lung Dis 2013; 17: 738-744.

83 Lempp JM, Zajdowicz MJ, Hankinson AL, et al. Assessment of the QuantiFERON-TB Gold In-Tube test for the detection of Mycobacterium tuberculosis infection in United States Navy recruits. PLoS One 2017; 12: e0177752.

84 Shrestha KB, Malla P, Jha KK, et al. First national tuberculin survey in Nepal. Int J Tuberc Lung Dis 2008; 12: 909-915.

85 Adetifa IMO, Muhammad AK, Jeffries D, et al. A tuberculin skin test survey and the annual risk of Mycobacterium tuberculosis infection in Gambian school children. PLoS One 2015; 10: e0139354.

86 Wassie L, Aseffa A, Abebe M, et al. Parasitic infection may be associated with discordant responses to QuantiFERON and tuberculin skin test in apparently healthy children and adolescents in a tuberculosis endemic setting, Ethiopia. BMC Infect Dis 2013; 13: 265.

87 Legesse M, Ameni G, Mamo G, et al. Community-based cross-sectional survey of latent tuberculosis infection in Afar pastoralists, Ethiopia, using QuantiFERON-TB Gold In-Tube and tuberculin skin test. BMC Infect Dis 2011; 11: 89.

88 Teklu T, Legesse M, Medhin G, et al. Latent tuberculosis infection and associated risk indicators in pastoral communities in southern Ethiopia: a community based cross-sectional study. BMC Public Health 2018; 18 : 266.

89 König Walles J, Tesfaye F, Jansson M, et al. Performance of QuantiFERON-TB Gold Plus for detection of latent tuberculosis infection in pregnant women living in a tuberculosis- and HIV-endemic setting. PLoS One 2018; 13: e0193589.

90 Lule SA, Mawa PA, Nkurunungi G, et al. Factors associated with tuberculosis infection, and with anti-mycobacterial immune responses, among five year olds BCG-immunised at birth in Entebbe, Uganda. Vaccine 2015; 33: 796-804.

91 Kizza FN, List J, Nkwata AK, et al. Prevalence of latent tuberculosis infection and associated risk factors in an urban African setting. BMC Infect Dis 2015; 15: 165.

92 Mumpe-Mwanja D, Verver S, Yeka A, et al. Prevalence and risk factors of latent tuberculosis among adolescents in rural Eastern Uganda. Afr Health Sci 2015; 15: 851-860.

93 Mathad JS, Bhosale R, Sangar V, et al. Pregnancy differentially impacts performance of latent tuberculosis diagnostics in a high-burden setting. PLoS One 2014; 9: e92308.

94 Gamsky TE, Lum T, Hung-Fan M, et al. Cumulative false-positive QuantiFERON-TB interferon- $\gamma$ release assay results. Ann Am Thorac Soc 2016; 13: 660-665.

95 Uppada DR, Selvam S, Jesuraj N, et al. The tuberculin skin test in school going adolescents in South India: associations of socio-demographic and clinical characteristics with TST positivity and non-response. BMC Infect Dis 2014; 14: 571 .

96 Thomas TA, Mondal D, Noor Z, et al. Malnutrition and helminth infection affect performance of an interferon $\gamma$-release assay. Pediatrics 2010; 126: e1522-e1529.

97 Hossain S, Zaman K, Banu S, et al. Tuberculin survey in Bangladesh, 2007-2009: prevalence of tuberculous infection and implications for TB control. Int J Tuberc Lung Dis 2013; 17: 1267-1272.

98 Raharimanga V, Ratovoson R, Ratsitorahina M, et al. Tuberculin reactivity in first-year schoolchildren in Madagascar. Trop Med Int Heal 2012; 17: 871-876.

99 Ranaivomanana P, Raharimanga V, Dubois PM, et al. Study of the BCG vaccine-induced cellular immune response in schoolchildren in Antananarivo, Madagascar. PLoS One 2015; 10: e0127590.

100 Sheriff FG, Manji KP, Manji MP, et al. Latent tuberculosis among pregnant mothers in a resource poor setting in Northern Tanzania: a cross-sectional study. BMC Infect Dis 2010; 10: 52.

101 Agaya J, Nnadi CD, Odhiambo J, et al. Tuberculosis and latent tuberculosis infection among healthcare workers in Kisumu, Kenya. Trop Med Int Health 2015; 20: 1797-1804.

102 Gustafson P, Lisse I, Gomes V, et al. Risk factors for positive tuberculin skin test in Guinea-Bissau. Epidemiology 2007; 18: 340-347.

103 Minime-Lingoupou F, Ouambita-Mabo R, Komangoya-Nzozo A-D, et al. Current tuberculin reactivity of schoolchildren in the Central African Republic. BMC Public Health 2015; 15: 496.

104 Mandalakas AM, Kirchner HL, Walzl G, et al. Optimizing the detection of recent tuberculosis infection in children in a high tuberculosis-HIV burden setting. Am J Respir Crit Care Med 2015; 191: 820-830.

105 Mancuso JD, Diffenderfer JM, Ghassemieh BJ, et al. The prevalence of latent tuberculosis infection in the United States. Am J Respir Crit Care Med 2016; 194: 501-509.

106 Mahomed H, Hawkridge T, Verver S, et al. Predictive factors for latent tuberculosis infection among adolescents in a high-burden area in South Africa. Int J Tuberc Lung Dis 2011; 15: 331-336. 
Mahomed H, Hughes EJ, Hawkridge T, et al. Comparison of Mantoux skin test with three generations of a whole blood IFN- $\gamma$ assay for tuberculosis infection. Int J Tuberc Lung Dis 2006; 10: 310-316.

108 Wood $\mathrm{R}$, Liang $\mathrm{H}$, Wu $\mathrm{H}$, et al. Changing prevalence of tuberculosis infection with increasing age in high-burden townships in South Africa. Int J Tuberc Lung Dis 2010; 14: 406-412.

109 Gallant CJ, Cobat A, Simkin L, et al. Tuberculin skin test and in vitro assays provide complementary measures of antimycobacterial immunity in children and adolescents. Chest 2010; 137: 1071-1077.

110 Lebina L, Abraham PM, Milovanovic M, et al. Latent tuberculous infection in schoolchildren and contact tracing in Matlosana, North West Province, South Africa. Int J Tuberc Lung Dis 2015; 19: 1290-1292.

111 Ncayiyana JR, Bassett J, West N, et al. Prevalence of latent tuberculosis infection and predictive factors in an urban informal settlement in Johannesburg, South Africa: a cross-sectional study. BMC Infect Dis 2016; 16 : 661.

112 Shanaube K, Sismanidis C, Ayles H, et al. Annual risk of tuberculous infection using different methods in communities with a high prevalence of TB and HIV in Zambia and South Africa. PLoS One 2009; 4: e7749.

113 Simpson T, Fox J, Crouse K, et al. Quantitative and qualitative QuantiFERON ${ }^{\circledR}$-TB Gold In-Tube results among groups with varying risks of exposure to tuberculosis. Heart Lung 2012; 41: 553-561.

114 Yang $\mathrm{X}, \mathrm{Wu} \mathrm{J}-\mathrm{B}$, Liu Y, et al. Identification of mycobacterial bacterioferritin B for immune screening of tuberculosis and latent tuberculosis infection. Tuberculosis 2017; 107: 119-125.

$115 \mathrm{Wu} \mathrm{X}$, Liang Y, Wang L, et al. Latent tuberculosis infection among new recruits to the army in Beijing, China in 2009. APMIS 2011; 119: 377-384.

116 Dheda K. Getting bang for buck in the latent tuberculosis care cascade. Lancet Infect Dis 2016; 16: 1209-1210.

117 O'Garra A, Redford PS, McNab FW, et al. The immune response in tuberculosis. Annu Rev Immunol 2013; 31: 475-527.

118 Diel R, Loddenkemper R, Nienhaus A. Predictive value of interferon- $\gamma$ release assays and tuberculin skin testing for progression from latent TB infection to disease state: a meta-analysis. Chest 2012; 142: 63-75.

119 Petruccioli E, Chiacchio T, Pepponi I, et al. First characterization of the CD4 and CD8 T-cell responses to QuantiFERON-TB Plus. J Infect 2016; 73: 588-597.

120 Theel ES, Hilgart H, Breen-Lyles M, et al. Comparison of the QuantiFERON-TB Gold Plus and QuantiFERON-TB Gold In-Tube interferon gamma release assays in patients at risk for tuberculosis and in health care workers. J Clin Microbiol 2018; 56. e00614-18.

121 Pieterman ED, Liqui Lung FG, Verbon A, et al. A multicentre verification study of the QuantiFERON ${ }^{\oplus}-$ TB Gold $^{-1}$ Plus assay. Tuberculosis 2018; 108: 136-142.

122 Lewinsohn DM, Leonard MK, LoBue PA, et al. Official American Thoracic Society/Infectious Diseases Society of America/Centers for Disease Control and Prevention clinical practice guidelines: diagnosis of tuberculosis in adults and children. Clin Infect Dis 2017; 64: e1-e33.

123 Pai M, Denkinger CM, Kik S V, et al. Gamma interferon release assays for detection of Mycobacterium tuberculosis infection. Clin Microbiol Rev 2014; 27: 3-20.

124 Jonsson J, Westman A, Bruchfeld J, et al. A borderline range for Quantiferon Gold In-Tube results. PLoS One 2017; 12: e0187313.

125 Farhat M, Greenaway C, Pai M, et al. False-positive tuberculin skin tests: what is the absolute effect of BCG and non-tuberculous mycobacteria? Int J Tuberc Lung Dis 2006; 10: 1192-1204.

126 Dale KD, Trauer JM, Dodd PJ, et al. Estimating the prevalence of latent tuberculosis in a low-incidence setting: Australia. Eur Respir J 2018; 52: 1801218. 